\title{
Recursos alimentares explorados pelos cupins (Insecta: Isoptera)
}

\author{
Juliana Toledo Lima ${ }^{1,2}$ \& Ana Maria Costa-Leonardo ${ }^{1}$
}

Biota Neotropica $v 7(n 2)$

http://www.biotaneotropica.org.br/v7n2/pt/abstract?thematic-review + bn04007022007

Recebido em 08/02/07

Versão Reformulada recebida em 21/05/07

Publicado em 11/07/07

${ }^{1}$ Departamento de Biologia, Instituto de Biociências, Universidade Estadual Paulista-UNESP
Avenida 24-A, 1515, Bela Vista, CP 199, CEP 13506-900, Rio Claro, SP, Brasil
${ }^{2}$ Autor para correspondência: Juliana Toledo Lima, e-mail: jtlima@ rc.unesp.br, www.rc.unesp.br

\begin{abstract}
Lima, J.T \& Costa-Leonardo, A.M. Food resources exploited by termites (Insecta: Isoptera). Biota Neotrop. May/Aug 2007 vol. 7, no. 2. http://www.biotaneotropica.org.br/v7n2/pt/abstract?thematic-review+bn04007022007. ISSN 1676-0603.

Termites are social insects belonging to the order Isoptera and constitute one of the dominant groups of invertebrates in terrestrial environments of the tropical region. Currently, there are around 2,900 species described in seven families: Mastotermitidae, Kalotermitidae, Termopsidae, Hodotermitidae, Serritermitidae, Rhinotermitidae and Termitidae. The Neotropical region contains 537 species, and from these, approximately 300 occur in Brazil and belong to the families Kalotermitidae, Rhinotermitidae, Serritermitidae and Termitidae. Although the Isoptera has been known by their potential as pest, the ecological role of the termites in the environment is primordial since they play a role as primary consumers and/or decomposers in natural ecosystems. These insects participate actively in the crushing, decomposition, humus production and mineralization of a variety of cellulose-based resources. A great diversity of organic material, in several stages of decomposition, may serve as food for termites, including wood (sound or decayed), grass, herbaceous plants, litter, fungi, nests built by other termite species, dung and carrion, lichen and even organic material present in the soil (humus). This vast range of food sources allowed the termites to occupy almost all the tropical and temperate regions of the Earth, occurring practically in all the terrestrial environments, natural or modified by the human species. Therefore, termites are found in the tropical and temperate forests, cerrados, savannas, caatingas, shallow places, mangrove regions, low vegetation stems, crops, pastures and urban environments.
\end{abstract}

Keywords: feeding habits, nourishment, food behavior, types of food, feeding-preference.

Resumo

Lima, J.T \& Costa-Leonardo, A.M. Recursos alimentares explorados pelos cupins (Insecta: Isoptera). Biota Neotrop. May/Aug 2007 vol. 7, no. 2. http://www.biotaneotropica.org.br/v7n2/pt/abstract?thematicreview+bn04007022007. ISSN 1676-0603.

Os cupins são insetos sociais pertencentes à ordem Isoptera e constituem um dos grupos de invertebrados dominantes em ambientes terrestres tropicais. Atualmente, existem cerca de 2.900 espécies descritas, que estão distribuídas em sete famílias: Mastotermitidae, Kalotermitidae, Termopsidae, Hodotermitidae, Serritermitidae, Rhinotermitidae e Termitidae. A região neotropical engloba 537 espécies, e dessas, aproximadamente 300 ocorrem no Brasil e pertencem às famílias Kalotermitidae, Rhinotermitidae, Serritermitidae e Termitidae. Apesar dos isópteros serem bastante conhecidos pelo seu potencial como praga, o papel ecológico dos térmitas no ambiente é primordial, visto que desempenham o papel de consumidores primários e/ou decompositores nos ecossistemas naturais. Esses insetos participam ativamente na trituração, decomposição, humificação e mineralização de uma variedade de recursos celulósicos. Uma grande diversidade de material orgânico, em vários estágios de decomposição, pode servir de alimento para os cupins, incluindo madeira (viva ou morta), gramíneas, plantas herbáceas, serapilheira, fungos, ninhos construídos por outras espécies de cupins, excrementos e carcaças de animais, liquens e até mesmo material orgânico presente no solo (húmus). Essa vasta gama de fontes alimentares permitiu aos cupins ocuparem quase todas as regiões quentes e temperadas da Terra, ocorrendo em praticamente todos os ambientes terrestres, naturais ou modificados pela espécie humana. Portanto, os isópteros são encontrados nas matas tropicais e temperadas, cerrados, savanas, caatingas, restingas, mangues, campos, culturas, pastagens e cidades.

Palavras-chave: hábitos alimentares, nutrição, comportamento alimentar, tipos de alimento, preferência alimentar. 


\section{Introdução}

A ordem Isoptera engloba os indivíduos conhecidos como cupins ou térmitas, os quais possuem um aparelho bucal do tipo mastigador (Grassé 1949). Atualmente, há 2.858 espécies descritas, sendo que a região neotropical engloba 537 espécies (Constantino 2007). Existem sete famílias de Isoptera: Mastotermitidae, Kalotermitidae, Termopsidae, Hodotermitidae, Serritermitidae, Rhinotermitidae e Termitidae (Grassé 1986). Os cupins pertencentes às seis primeiras famílias são denominados "cupins inferiores" e os pertencentes à sétima família (Termitidae) são denominados "cupins superiores". Tais designações não significam que os insetos apresentam similaridades filogenéticas nem diferentes origens, referem-se apenas à dependência de protozoários flagelados simbiônticos, no caso dos "térmitas inferiores”, para auxiliar na degradação da celulose (Costa-Leonardo 2002). No Brasil ocorrem aproximadamente 300 espécies, que se distribuem entre as famílias Kalotermitidae, Rhinotermitidae, Serritermitidae e Termitidae (Constantino 1999).

Os cupins são os invertebrados dominantes em ambientes terrestres tropicais e estão espalhados desde as florestas úmidas até as savanas, sendo encontrados até mesmo em regiões áridas (Lee \& Wood 1971, La Fage \& Nutting 1978, Wood \& Sands 1978, Wood et al. 1982, Eggleton et al. 1996). A explicação para essa extraordinária abundância advém da existência da simbiose com microorganismos, além de uma organização social bastante desenvolvida (Costa-Leonardo 2002). A ordem Isoptera é bastante conhecida pelo seu potencial como praga, apesar dos cupins-praga constituírem a minoria dentro do grupo (cerca de 10\%). Contudo, o papel ecológico desses insetos no ambiente é primordial, uma vez que ocupam a posição de consumidores primários ou decompositores (herbívoros e detritívoros) nos ecossistemas naturais, atuando na reciclagem de nutrientes por meio da trituração, decomposição, humificação e mineralização de uma variedade de recursos celulósicos (Tayasu et al. 1997, Costa-Leonardo 2002). Geralmente assume-se que todos os cupins são consumidores de madeira (xilófagos), porém uma grande diversidade de material orgânico, em vários estágios de decomposição, pode servir de alimento para esses insetos, incluindo madeira (viva ou morta), gramíneas, plantas herbáceas, serapilheira, fungos, ninhos construídos por outras espécies de cupins, excrementos e carcaças de animais, liquens e até mesmo material orgânico presente no solo (Lee \& Wood 1971, La Fage \& Nutting 1978, Wood 1978, Noirot 1992, Sleaford et al. 1996, Miura \& Matsumoto 1997, Donovan et al. 2001).

$\mathrm{Na}$ sociedade dos cupins existem indivíduos morfofisiologicamente diferentes, que compreendem as diferentes castas, responsáveis por diferentes tarefas. A casta estéril é representada pelos operários e soldados, sendo que os primeiros são os responsáveis pela busca e consumo imediato do alimento. Já os soldados atuam na defesa, enquanto a casta fértil, representada pelo casal real (rei e rainha), está envolvida nas atividades reprodutivas (Krishna 1969).

A biologia alimentar é o fator que afeta mais significativamente a história de vida e a evolução social dos Isoptera. A ecologia nutricional dos cupins é considerada fundamental para o princípio da eussocialidade e influencia de maneira bastante consistente e penetrante em virtualmente todos os aspectos da gênese de diversificação desse grupo e da evolução da eussocialidade (Abe 1987, Noirot 1992, Higashi et al. 1992, Bignell 1994, Nalepa 1994, Thorne 1997). A dieta de madeira dos cupins demandou uma dependência coevolutiva de simbiontes do trato digestivo e de suas habilidades celulolíticas (Cleveland et al. 1934), que "fizeram com que a sobreposição de gerações se tornasse uma necessidade" (Thorne 1997). A chave evolutiva e os elementos ecológicos da biologia dos cupins, tais como o tamanho da colônia, a história de vida, o desenvolvimento de castas e os habitats (ninhos), estão associados à disponibilidade de recursos alimentares (Abe 1987, Lenz 1994).

\section{Tipos de Alimentação}

Existem diferentes tipos de alimentação entre os cupins. Os jovens, os soldados e todos os reprodutores são incapazes de se alimentar sozinhos e recebem dos operários alimentação estomodeal ou proctodeal. A alimentação estomodeal pode ser saliva, que é o único nutriente dos reprodutores funcionais (rei e rainha), ou alimento regurgitado. Os soldados são, em grande parte, nutridos com alimento regurgitado, mas em certos Termitidae eles têm uma dieta exclusivamente líquida (saliva). A alimentação proctodeal foi descrita para os cupins inferiores, os quais apresentam uma fauna intestinal composta por protozoários flagelados. $\mathrm{O}$ alimento proctodeal consiste de excreções líquidas, ricas em simbiontes, provenientes do intestino posterior e que são eliminadas em resposta a estímulos táteis de outros cupins. Na família Kalotermitidae este alimento é totalmente distinto das fezes sólidas e secas do reto (Grassé 1949).

\section{Categorias de Recursos Alimentares Explorados pelos Cupins}

A coleta de alimento pelos operários, tanto para uso próprio quanto para prover as castas dependentes, é o recurso energético básico da colônia. Ela consiste de materiais celulósicos provenientes de plantas vivas ou mortas, parcialmente ou quase que inteiramente decompostas. O recurso alimentar e sua subseqüente decomposição têm muitas implicações de longo alcance nas relações entre cupins e solos, tanto por suas atividades alimentares quanto pela transformação do alimento por meio da digestão. Portanto, os cupins afetam o ciclo da matéria orgânica e dos nutrientes. Adicionalmente, a concentração de alimento e a perda de produtos a partir da digestão influenciam a disposição de matéria orgânica e de nutrientes no ecossistema (Lee \& Wood 1971).

Os térmitas exploram alimentos com baixo valor nutricional se comparados àqueles consumidos por outros animais. A capacidade de sobreviver a partir da ingestão de alimentos pobres em nutrientes se deve aos mecanismos digestivos usados para extrair a maior parte dos nutrientes disponíveis a partir de uma alimentação de baixa qualidade, à conservação altamente controlada desses recursos e à ciclagem de nutrientes (Waller \& La Fage 1986). Os diferentes tipos de recursos alimentares apresentam problemas e benefícios especiais para as sociedades de cupins, e esses aspectos serão discutidos a seguir.

\section{Madeira}

Grassé \& Noirot (1959) consideram a dieta xilófaga como primitiva. Essa dieta é mantida não somente pela maioria dos cupins inferiores, mas também por quase todos os Termitidae, com exceção dos representantes da subfamília Apicotermitinae (Bignell \& Eggleton 2000). Contudo, a condição da madeira (viva ou morta, sã ou decomposta) parece ser importante para a determinação se ela é ou não adequada como recurso alimentar para as espécies de cupins.

Madeira viva é usada principalmente pelos cupins inferiores, incluindo espécies de Zootermopsis (família Termopsidae), Heterotermes, Schedorhinotermes, Coptotermes, Reticulitermes (família Rhinotermitidae) e membros das famílias Kalotermitidae e Mastotermitidae, e é raramente consumida pelas espécies pertencentes à família Termitidae (Lee \& Wood 1971; Wood 1978). Em geral, consumidores de madeira viva se alimentam apenas de porções mortas de árvores sadias, incluindo cerne e troncos mortos. Além de terem baixo valor nutritivo e conteúdo tóxico potencialmente alto (Anderson 1962), a madeira é dura e pode causar um desgaste con- 
siderável nas mandíbulas dos cupins, embora nenhum estudo tenha ainda mencionado tal fato. Desse modo, muitas espécies de árvores são indisponíveis para alguns cupins (Scheffrahn \& Rust 1983). Além disso, há muitas variações intra-específicas na distribuição radial e vertical de toxinas (Anderson 1962), bem como na quantidade de substâncias tóxicas presentes (Labosky Jr. 1979), dificultando ainda mais o consumo por parte dos térmitas. As vantagens do consumo de árvores vivas incluem um ambiente de forrageamento abrigado, que está isolado de temperaturas e umidades extremas (Highley \& Kirk 1979).

Os cupins consomem madeira morta sã em vários estágios de decomposição. Esse recurso alimentar é consumido tanto pelos cupins inferiores como pelos superiores, e mostra as mesmas desvantagens da madeira viva no baixo conteúdo de nitrogênio, na dureza e no potencial tóxico elevado. Adicionalmente, amplas flutuações de temperatura e umidade ocorrem na madeira cortada (Highley \& Kirk 1979). Além disso, esse tipo de madeira pode abrigar formigas predadoras. Madeira recém-abatida é a dieta primordial dos Kalotermitidae, Rhinotermitidae e certos membros de Hodotermitidae e Termitidae. Entre os cupins superiores, pertencentes à família Termitidae, muitos dos Macrotermitinae e Nasutitermitinae se alimentam desse substrato. Já madeira decomposta ou envelhecida é a principal fonte alimentar de muitos Hodotermitidae (todas as subfamílias, exceto Hodotermitinae), Rhinotermitidae, certos membros de Termitidae e poucos Kalotermitidae.

Fungos decompositores podem transformar alimentos pobres em palatáveis ou alimentos bons em impalatáveis, dependendo do fungo, do cupim e do alimento hospedeiro (Sands 1969, La Fage \& Nutting 1978, Amburgey 1979; Gilbertson 1984). As vantagens potenciais de comer alimentos decompostos incluem: 1 . aumento da quantidade de nitrogênio e de outros nutrientes; 2 . aumento no conteúdo de umidade; 3. pré-digestão de produtos da madeira; 4 . aquisição de bactérias fixadoras de nitrogênio, comumente associadas às raízes; 5 . acesso a nutrientes ricos em esporóforos fungais; e 6. desintoxicação da madeira e de extratos de plantas (Martin 1979).

Muitos cupins só se alimentam após a madeira atingir um estado avançado de decomposição, e é possível que os fungos sejam um importante constituinte na dieta dessas espécies. Lenz (1976) verificou aumento na reprodução de Heterotermes alimentado por madeira envelhecida, já decomposta. A modificação por decomposição pode ser relevante para muitas espécies que vivem no cerne das árvores, já que essa área funciona como um local de depósito de toxinas das plantas (Highley \& Kirk 1979). O estágio de decomposição é importante possivelmente por causa das mudanças nas taxas de carbono-nitrogênio presentes na fonte celulósica. Por isso, madeira sã geralmente não é atacada pelos cupins, e madeiras em estágios avançados de decomposição podem até ser evitadas por esses insetos (La Fage \& Nutting 1978). Muitos cupins parecem ser nutricionalmente dependentes de certos fungos decompositores de madeira. Outros, embora não dependentes dos fungos, se beneficiam da sua presença, e muitas espécies (incluindo alguns dos consumidores de madeiras tipicamente sãs, recém-abatidas, tais como Kalotermitidae) mostraram uma resposta positiva para madeira infectada com fungo ou para os extratos fungais; no entanto, algumas espécies mostraram resposta negativa (Sands 1969). Grassé (1949) e outros pesquisadores sugeriram que tais fungos provêm vitaminas e "fatores de crescimento", mas isso não foi demonstrado empiricamente.

Algumas das espécies de cupins que se alimentam de madeira tendem a ser polífagas e não consomem somente esse recurso, mas uma grande variedade de plantas vivas, fragmentos de plantas, esterco, além de muitos produtos armazenados e materiais usados pelo homem. Esses térmitas polífagos são as maiores pragas em muitas áreas (Snyder 1948; Ratcliffe et al. 1952, Harris 1961, 1970).
Mesmo em um determinado pedaço de madeira os cupins podem selecionar certas porções em preferência a outras (Snyder 1948; Bouilon 1970). Rudman \& Gay (1963, 1967a, b) investigaram alguns dos fatores responsáveis pela resistência natural de certas árvores ao ataque de cupins. Eles concluíram que as substâncias químicas presentes na madeira foram o principal fator de resistência e de diferenças na suscetibilidade de certas espécies em regiões diferentes.

\section{Grama, plantas herbáceas e serapilheira}

Grama, plantas herbáceas e serapilheira podem ser usadas diretamente como alimento pelos cupins ou como substratos para favos de fungos de térmitas da subfamília Macrotermitinae (cupins que cultivam fungos). Esses recursos requerem forrageamento por grandes áreas (Darlington 1982). Além de se mostrarem potencialmente competitivos em relação aos outros herbívoros (Collins 1982), os cupins "coletores" ("harvesters") devem dominar os aleloquímicos presentes na grama fresca (Botha \& Hewitt 1978). Esses problemas podem ser superados pela vantagem do alto conteúdo de nitrogênio na grama em relação à madeira (La Fage \& Nutting 1978) e também pelo acesso ao esterco produzido pelos mamíferos herbívoros (Ferrar \& Watson 1970).

Os cupins "coletores" ("harvesters") incluem espécies que forrageiam na superfície e cortam gramas, plantas herbáceas, folhas, galhos, sementes e outras partes de plantas depositadas na superfície do solo. Madeira e serapilheira variam em conteúdo de nitrogênio num determinado habitat (Prestwich et al. 1980), e podem ser consumidas em vários estágios de decomposição (Wood 1978). Cupins que consomem grama, geralmente morta, são encontrados nas subfamílias Macrotermitinae, Termitinae e Nasutitermitinae, pertencentes à família Termitidae, e nos Hodotermitinae (família Hodotermitidae) (Bignell \& Eggleton 2000). De acordo com os mesmos autores, serapilheira, na forma de galhos, ramos e folhas, é consumida pelos cupins das subfamílias Macrotermitinae, Apicotermitinae, Termitinae e Nasutitermitinae (família Termitidae).

Os verdadeiros cupins coletores ("harvesters") são os Hodotermitinae, família Hodotermitidae (Hodotermes da África, Microhodotermes do Norte da África e Anacanthotermes do Norte da África e da faixa árida da Ásia); alguns Amitermitinae, família Termitidae (tais como Drepanotermes na Austrália); e alguns Nasutitermitinae, família Termitidae (tais como certos Nasutitermes e Tumulitermes na Austrália, Trinervitermes na África e Indo-Malásia e Syntermes na América do Sul). Essas espécies invariavelmente acumulam o material coletado em seus ninhos, algumas vezes em quantidades extremamente grandes (Lee \& Wood 1971). O gênero Syntermes é exclusivamente neotropical (Constantino 1995) e destaca-se como um dos poucos consumidores de folhas da serapilheira (Bandeira 1991). Esses cupins contribuem para a decomposição de várias maneiras: por meio do consumo direto e posterior digestão, pela fragmentação das folhas da serapilheira, aumentando assim a disponibilidade desse material para outros consumidores, e pela adição do material cortado e abandonado na superfície ou no perfil do solo (Medeiros 2001). Os Syntermes geralmente são ativos à noite, apesar de eventualmente serem encontrados forrageando durante o dia, quando o tempo está nublado (Mill 1984). De acordo com Barbosa (1993), o horário de maior atividade de $S$. spinosus e $S$. molestus está entre 19:00 e 02:00 horas. Eles cortam pedaços de folhas presentes na superfície do solo, as quais podem ser recém-caídas ou envelhecidas, e os carregam para seus ninhos. Os gêneros neotropicais Velocitermes, Rhynchotermes e Cornitermes (família Termitidae, subfamília Nasutitermitinae) também se enquadram nesse grupo consumidor de serapilheira (Constantino 2005, Costa-Leonardo 2005). No Brasil, Cornitermes cumulans, uma espécie de cupim de montículo bastante conhecida pela alta incidência em pastagens, se alimenta principal- 
mente de folhas e raízes mortas de gramíneas. No ninho (Figuras 1a e 1b), os alimentos são mastigados e posteriormente armazenados nas paredes das câmaras do cupinzeiro (Figuras 1c e 1d).

Os operários do gênero neotropical Ruptitermes (família Termitidae, subfamília Apicotermitinae) coletam fragmentos de folhas e sementes na superfície do solo e não consomem imediatamente esse alimento, que é armazenado em galerias subterrâneas esparsas, formando o seu ninho "difuso". Eles também podem ocupar ninhos epígeos construídos por outras espécies e estocar alimento nas galerias do referido montículo abandonado (Costa-Leonardo 2006).

\section{Fungos}

A associação entre cupins e fungos alcançou seu mais alto nível de especialização na subfamília Macrotermitinae (família Termitidae), que ocorre na África tropical, em partes da Índia e da Arábia, e no sudeste asiático, e geralmente são os cupins dominantes das savanas e de florestas tropicais desses lugares. Os Macrotermitinae consomem madeira, folhas e grama morta, fezes de herbívoros, e podem também se alimentar de raízes. Algumas espécies armazenam alimento na forma de minúsculos fragmentos de madeira umedecidos com saliva. Depois de ser estocado por uma semana, o alimento é ingerido e então depositado como fezes nos favos de fungos (Wood \& Thomas 1989, Darlington 1994).

Os membros dessa subfamília cultivam o basidiomiceto Termitomyces, pertencente à família Amanitaceae, em um substrato de fezes produzido a partir da ingestão de alimento, que passa rapidamente pelo trato digestivo do cupim, sem sofrer digestão, e é expelido sob a forma de esferas (fezes primárias). Em Macrotermes e Odontotermes o fungo fica no centro das câmaras de cria, porém, várias espécies subterrâneas não apresentam uma câmara central, sendo que o fungo se aloja em pequenas câmaras espalhadas pelo solo, em profundidades variadas. Os favos de fungos são estruturas bastante dinâmicas, e conforme as fezes são depositadas e o fungo cresce, a parte mais antiga é consumida pelos cupins, resultando numa "reciclagem" total num período de cinco a oito semanas (Wood \& Thomas 1989, Darlington 1994). Os fungos são ricos em nitrogênio e outros nutrientes, além de processarem enzimas (celulases, amilase, sucrase, maltase, melibiase, celobiase, lactase, xilanase, galactanase, proteinase, polipeptidase e lipase) para o catabolismo de celulose e lignina. Os favos de fungos também podem desintoxicar aleloquímicos (Martin \& Martin 1979). $\mathrm{O}$ cultivo de jardins de fungos otimiza a assimilação do alimento, e seus cultivadores apresentam taxas de consumo muito mais altas do que outros consumidores (Collins 1980).

A existência desse mutualismo entre cupins e fungos proporcionou aos térmitas um aproveitamento mais eficiente de nitrogênio e energia, sem serem limitados pela quantidade de simbiontes que seu intestino pode comportar. Enquanto os cupins inferiores precisam carregar consigo verdadeiras câmaras de fermentação para digerirem a madeira, os Macrotermitinae simplesmente deixam o fungo fazer esse trabalho para eles, possibilitando que os operários sejam mais leves e ativos. É possível também que esses cupins possam ser capazes de aproveitar materiais vegetais que seriam impossíveis de serem digeridos sem o fungo. Já o fungo, por sua vez, tem cerca de $25 \%$ do material vegetal disponível já cortado em pedacinhos, umedecido e trazido até ele, num ambiente de temperatura e umidade constantes, onde suas hifas podem penetrar
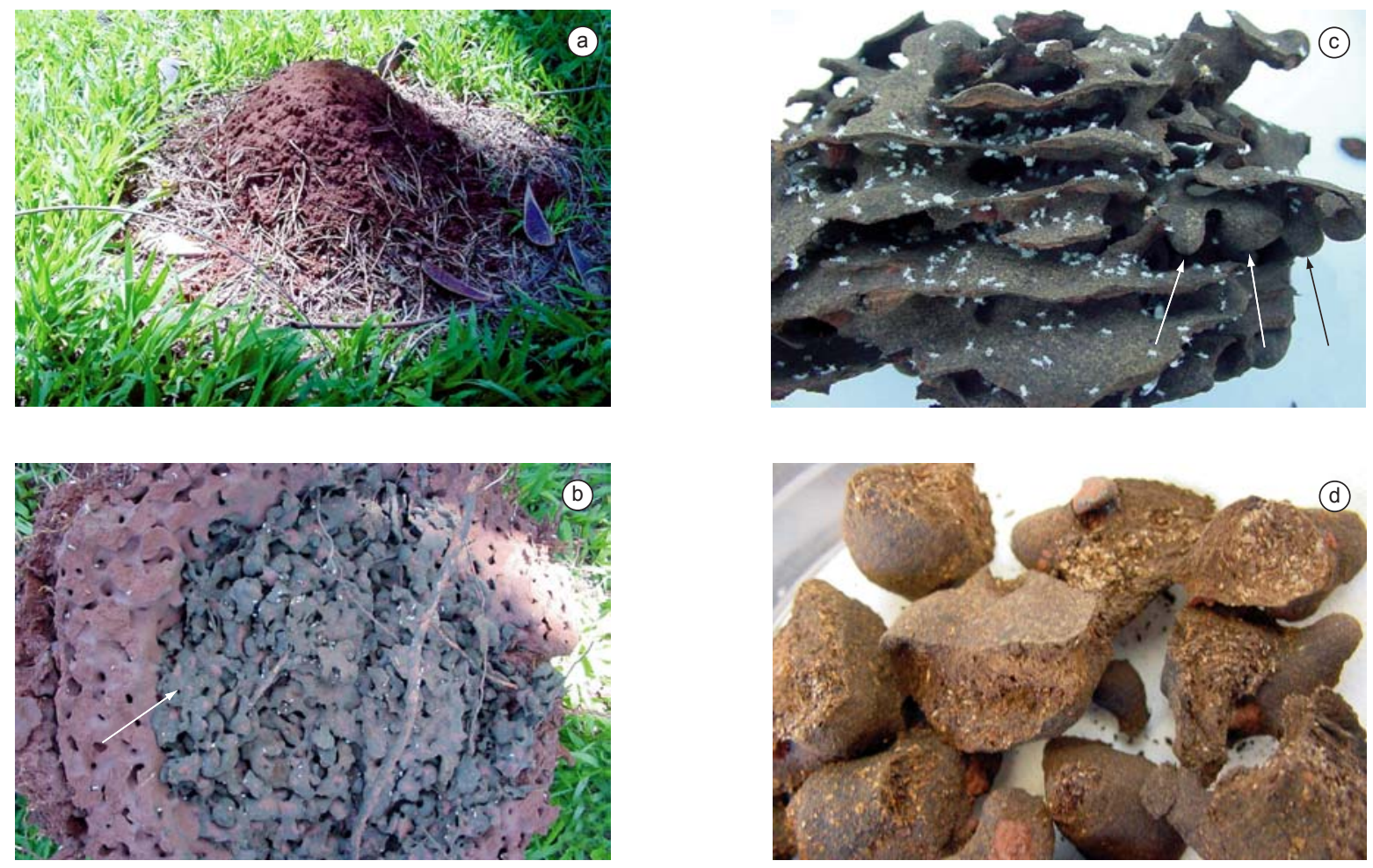

Figura 1. Ninho do cupim Cornitermes cumulans. a) Vista geral externa; b) Ninho em corte transversal evidenciando o núcleo interno celulósico (seta) e a parede externa argilosa; c) Detalhe do núcleo interno com as câmaras de alimento (setas); e d) Detalhe de algumas câmaras de alimento isoladas do núcleo interno do ninho.

Figure 1. Mound of the termite Cornitermes cumulans. a) General external view; b) Transversal section of the mound showing the internal cellulosic nucleus (arrow) and the external wall of clay; c) Detail of the internal nucleus with the food chambers (arrows); and d) Detail of some food chambers which were isolated from the internal nucleus of the mound. 
rapidamente e seus competidores são suprimidos (Wood \& Thomas 1989, Darlington 1994).

\section{Produtos Animais}

Os cupins ingerem produtos animais na forma de fezes (ninhos cartonados construídos por outras espécies de cupins e excrementos de mamíferos) e carcaças (La Fage \& Nutting 1978, Wood 1978).

\subsection{Ninho cartonado}

Alguns cupins constroem ninhos cartonados que são consumidos por outras espécies de térmitas que coabitam no mesmo cupinzeiro, denominadas inquilinas (Mathews 1977). Esse material cartonado é composto por fezes, saliva e solo, e compreende um recurso alimentar abundante porque é continuamente renovado pelos cupins hospedeiros. Além de servir como abrigo para os inquilinos, esses ninhos possuem uma alta capacidade de manutenção de umidade (Lee \& Wood 1971). Mathews (1977) e Constantino (2005) relatam que espécies do gênero Inquilinitermes, cupins que vivem exclusivamente em ninhos de Constrictotermes, se alimentam do material fecal acumulado na base desses ninhos.

\subsection{Esterco}

O esterco é rico em nitrogênio (La Fage 1976) e materiais celulósicos. Os cupins subterrâneos são os decompositores primários desse tipo de recurso alimentar em determinados habitats desérticos (Whitford et al. 1982). Alguns cupins consumidores de grama e serapilheira usam excrementos animais como alimento suplementar. Contudo, Ferrar \& Watson (1970) relataram que algumas espécies australianas consomem exclusivamente esterco, principalmente de origem bovina. $\mathrm{O}$ alto conteúdo de umidade pode ser importante para alguns consumidores desse tipo de recurso alimentar (Collins 1983), porém, Coe (1977) verificou que a água não foi o atrativo primário em estrume de elefante, e, de acordo com Ettershank et al. (1980), os cupins do deserto evitam o consumo de esterco úmido. A ocorrência de excrementos animais é irregular, tanto espacialmente como sazonalmente (Whitford et al. 1982), e a predação por aves e formigas (Coe 1977) está associada a essas espécies de cupim que consomem esterco.

\subsection{Carcaças}

Thorne \& Kimsey (1983) observaram cupins do gênero Nasutitermes consumindo carcaças (ossos, pele, órgãos internos e musculatura) decompostas de animais numa floresta tropical do Panamá (Barro Colorado Island) em períodos de seca, sendo que nenhum inseto saprófago foi encontrado após a ocorrência de chuva. O consumo desse recurso alimentar por Nasutitermes requer clima seco para localização e acesso ao corpo do animal. Além disso, a atração por carcaças na estação seca deve refletir as necessidades nutricionais desses cupins, uma vez que as secas precedem a formação dos indivíduos alados, que atuarão na dispersão da espécie nos períodos chuvosos. De acordo com Thorne \& Kimsey (1983) a demanda de nitrogênio dos cupins não é facilmente suprida com uma dieta basicamente celulósica, e muitas espécies eliminam tal carência por meio de ajustes comportamentais ou fisiológicos, explicando o consumo dessas carcaças animais.

\section{Húmus}

Aproximadamente $60 \%$ dos Termitidae, incluindo os Apicotermitinae, a maioria dos Termitinae, e alguns Nasutitermitinae, têm se especializado convergentemente no consumo de solo (Anderson \& Wood 1984, Bignell \& Eggleton 2000). Húmus consiste de minerais orgânicos, carboidratos, micróbios do solo, compostos polifenólicos e também hexosaminas, que constituem 5-13\% dos solos e derivam dos restos de artrópodos e fungos (Collins 1983). Não se sabe quais nutrientes do solo são extraídos pelos humívoros, mas o alongamento do intestino desses cupins (Bignell et al. 1983) sugere uma dieta de baixa qualidade. Comedores de solo estão restritos aos habitats com alta umidade, tais como florestas úmidas e savanas, e são sensíveis aos distúrbios ambientais (Wood et al. 1982).

Os cupins humívoros apresentam adaptações nas suas mandíbulas, as quais perderam os sulcos transversais existentes nas placas molares e ficaram com a borda mais arredondada (Sands 1965, 1972). Além das modificações nas peças bucais, a espécie brasileira nativa Tetimatermes oliveirae apresenta a tíbia anterior muito dilatada, com uma cavidade bastante desenvolvida (semelhante a uma colher), que é adaptada para escavação (Fontes 1992). Essas espécies humívoras parecem ser amplamente distribuídas por toda a região neotropical, Etiópia e regiões orientais (Araújo 1970, Bouillon 1970, Roonwall 1970). De acordo com Constantino (2005), os cupins humívoros que vivem em ambientes de cerrado compreendem o grupo mais diversificado, e engloba quase todos as espécies pertencentes à subfamília Apicotermitinae (família Termitidae).

\section{Dietas Especiais}

\subsection{Canibalismo, necrofagia e oofagia}

Registros de canibalismo são comuns em estudos laboratoriais (La Fage \& Nutting, 1978), mas o impacto desse comportamento na natureza é incerto. O canibalismo parece ser uma resposta à falta de proteína sob determinadas circunstâncias. Revisões de Le \& Wood (1971) e Collins (1983) sugeriram que os cupins recorrem ao canibalismo sob condições de estresse, tais como a carência de nitrogênio. A hemolinfa dos indivíduos injuriados estimula o canibalismo, e os ácidos graxos promovem a necrofagia. A função primária do canibalismo é provavelmente a de higiene do cupinzeiro, visto que relativamente poucas espécies matam cupins do seu próprio ninho. A conservação de proteína pode ter uma importância secundária, mas pode ocasionalmente contribuir para a sobrevivência da colônia (Collins, 1983).

Grassé (1949) notou que nos primeiros estágios da fundação da colônia o rei e a rainha geralmente consomem uma considerável quantidade de ovos (oofagia). Adicionalmente, os operários também consomem indivíduos doentes e feridos, cadáveres e exúvias. Muitos autores notaram que os operários podem controlar a proporção das várias castas por meio do canibalismo seletivo (Lee \& Wood 1971, Collins 1983). Necrofagia na mesma casta também ocorre. Batra \& Batra (1977) reportaram que rainhas mortas de Odontotermes são comidas, possivelmente por causa do seu alto valor nutritivo, ao passo que outras castas são enterradas. Em algumas espécies de cupins, machos e fêmeas que perderam recentemente suas asas mordem as extremidades das antenas um do outro como um prelúdio à fundação da colônia. Esse "canibalismo" foi previamente sugerido como resultado da nutrição inadequada, mas atualmente acredita-se que seja uma mudança de comportamento de orientação prévia dos alados para então iniciarem a cópula (Hewitt et al. 1972).

\subsection{Liquens}

Espécies de cupins do gênero Hospitalitermes, pertencentes à subfamília Nasutitermitinae (família Termitidae), que ocorrem no sudeste da Ásia, forrageiam "a céu aberto" em colunas de troncos e nas copas das árvores das florestas tropicais (Kalshoven 1958, Collins 1979, Jones \& Gathorne-Hardy 1995, Miura \& Matsumoto 1997, 1998). Sua dieta alimentar é composta por madeira, briófitas, algas azuis, hifas de fungos e, em grande parte, por liquens coletados 
na superfície dos galhos de árvores (Collins 1979). Porém, não se sabe se são os fungos ou as algas, os quais compõem esses liquens, o recurso alimentar mais importante. Os operários transportam o material coletado, do local de forrageamento até o ninho, na forma de "bolotas alimentares", que são carregadas com o auxílio de suas mandíbulas (Miura \& Matsumoto 1997). Segundo os mesmos autores, essas bolotas são maiores que a cabeça dos operários de Hospitalitermes e são compostas por fibras de material vegetal. Essa estratégia de coleta de alimento é arriscada porque esses operários são expostos tanto a predação como a estresse ambiental, e uma vez que o benefício nutricional desse hábito alimentar deve superar o custo do forrageamento "a céu aberto", Collins (1979) sugeriu que as "bolotas alimentares" constituem uma fonte importante de nitrogênio para esses cupins.

\section{Considerações Finais}

A partir do exposto, pode-se constatar que existe uma ampla variedade de recursos alimentares que são explorados pelos Isoptera. Essa grande variedade permitiu aos cupins ocuparem quase todas as regiões quentes e temperadas da Terra, ocorrendo em praticamente todos os ambientes terrestres, naturais ou modificados pela espécie humana. Em vista do exposto, esses insetos são encontrados nas matas tropicais e temperadas, cerrados, savanas, caatingas, restingas, mangues, campos, culturas, pastagens e cidades (Fontes 1979).

\section{Agradecimentos}

Nós agradecemos à Fundação de Amparo à Pesquisa do Estado de São Paulo (FAPESP) pela bolsa de Mestrado (proc. 04/04859-7) e ao Conselho Nacional de Desenvolvimento Científico e Tecnológico $(\mathrm{CNPq})$ pela bolsa de Produtividade em Pesquisa (proc. 301980/2004-7).

\section{Referências Bibliográficas}

ABE, T. 1987. Evolution of the life types in termites. In Evolution, coadaptation, and biotic communities (S. Kawano, J.H. Connel \& T. Hidaka, eds.). University of Tokyo Press, Tokyo, p.128-148.

AMBURGEY, T.L. 1979. Review and checklist of the literature on interactions between wood-inhabiting fungi and subterranean termites: 1960-1978. Sociobiology. 4(2):279-296.

ANDERSON, A.B. 1962. On the chemistry of wood rot. Tappi 45(1):40-62.

ANDERSON, J.M. \& WOOD, T.G. 1984. Mound composition and soil modification by two soil-feeding termites (Termitinae, Termitidae) in a riparian Nigerian forest. Pedobiologia. 26(2):77-82.

ARAÚJO, R.L. 1970. Termites of the Neotropical region. In Biology of termites (K. Krishna \& F.M. Weesner, eds.). Academic Press, New York, v.2, p.527-576.

BANDEIRA, A.G. 1991.Térmitas (Insecta: Isoptera) consumidores de liteira na Ilha de Maracá, Roraima. Acta Amazon. 21(1):15-23.

BARBOSA, R.I. 1993. Período de forrageamento de duas espécies de Syntermes (Isoptera, Termitidae) em uma floresta tropical amazônica e a relação com temperatura e umidade do ar. Rev. Bras. Entomol. 37(4):763-767.

BATRA, L.R. \& BATRA, S.W.T. 1977. Termite-fungus mutualism. In Insectfungus symbiosis, nutrition, mutualism, and commensalism (L.R. Batra, ed.). John Wiley \& Sons, New York, p.117-163.

BIGNELL, D.E. 1994. Soil-feeding and gut morphology in higher termites. In Nourishment and evolution in insect societies (J.H. Hunt \& C.A. Nalepa, eds.). Westview Press, Boulder, p.131-159.

BIGNELL, D.E. \& EGGLETON, P. 2000. Termites in ecosystems. In Termites: evolution, sociality, symbioses, ecology (T. Abe, D.E. Bignell \& M. Higashi, eds.). Kluwer Academic Publishers, London, p.363-387.

BIGNELL, D.E., OSKARSSON, H., ANDERSON, J.M., INESON, P. \& WOOD, T.G. 1983. Structure, microbial associations and function of the so-called "mixed segment" of the gut in two soil-feeding termites, Procubitermes aburiensis and Cubitermes severus (Termitidae, Termitinae). J. Zool. 201(4):445-480.

BOTHA, T.C. \& HEWITT, P.H. 1978. Influence of diets containing green material on laboratory colonies of Hodotermes mossambicus (Hagen). Phytophylactica. 10:93-97.

BOUILLON, A. 1970. Termites of the Ethiopian region. In Biology of termites (K. Krishna \& F.M. Weesner, eds.). Academic Press, New York, v.2, p.153-280.

CLEVELAND, L., HALL, S.R., SANDERS, E.P. \& COLLIER, J. 1934. The wood-feeding roach Cryptocercus, its Protozoa, and the symbiosis between Protozoa and roach. Mem. Am. Acad. Arts Sci. 17:185-342.

COE, M. 1977. The role of termites in the removal of elephant dung in the Tsavo (East) National Park Kenya. East Afr. Wildl. J. 15(1):49-55.

COLLINS, N.M. 1979. Observations on the foraging activity of Hospitalitermes umbrinus (Haviland), (Isoptera: Termitidae) in the Gunong Mulu National Park, Sarawak. Ecol. Entomol. 4(3):231-238.

COLLINS, N.M. 1980. The effect of logging on termite (Isoptera) diversity and decomposition processes in lowland dipterocarp forests. In Tropical ecology and development (J. I. Furtado, ed.). International Society for Tropical Ecology, Kuala Lumpur, p.113-121.

COLLINS, N.M. 1982. The interaction and impact of domestic stock and termites in a Kenyan rangeland. In: The biology of social insects (M.D. Breed, C.D.Michener \& H.E. Evans, eds.). Westview Press, Boulder, p.80-84.

COLLINS, N.M. 1983. The utilization of nitrogen resources by termites (Isoptera). In Nitrogen as an ecological factor (J.A. Lee, S. McNeill \& I.H. Rorison, eds.). Oxford University Press, Oxford, p.381-412.

CONSTANTINO, R. 1995. Revision of the Neotropical genus Syntermes Holmgren (Isoptera: Termitidae). Un. Kan. Sci. Bull. 55(13):455-518.

CONSTANTINO, R. 1999. Chave ilustrada para identificação dos gêneros de cupins (Insecta: Isoptera) que ocorrem no Brasil. Pap. Avulsos Zool. 40(25):387-448

CONSTANTINO, R. 2005. Padrões de diversidade e endemismo de térmitas no bioma Cerrado. In Cerrado: ecologia, biodiversidade e conservação (A. Scariot, J.C. Sousa-Silva \& J.M. Felfili, orgs.). Ministério do Meio Ambiente, Brasília, p.320-333.

CONSTANTINO, R. 2007. On-Line Termites Database. (Disponível em: http://www.unb.br/ib/zoo/docente/constant/catal/catnew.html). Acesso em: 4. jan. 2007.

COSTA-LEONARDO, A.M. 2002. Cupins-praga: morfologia, biologia e controle. Ana Maria Costa-Leonardo, Rio Claro.

COSTA-LEONARDO, A.M. 2005. Arquitetos das pastagens e beiras de estradas. Cienc. Hoje. 36(216):62-65.

COSTA-LEONARDO, A.M. 2006. Os cupins "kamikazes". Cienc. Hoje 38(225):68-70.

DARLINGTON, J.P.E.C. 1982. The underground passages and storage pits used in foraging by a nest of the termite Macrotermes michaelseni in Kajiado, Kenya. J. Zool. 198(2):237-247.

DARLINGTON, J.P.E.C. 1994. Nutritional and evolution in fungus-growing termites. In Nourishment and evolution in insect societies (J.H. Hunt \& C.A. Nalepa, eds.). Westview Press, Boulder, p.105-130.

DONOVAN, S. E., EGGLETON, P. \& BIGNELL, D.E. 2001. Gut content analysis and a new feeding group classification of termites. Ecol. Entomol. 26(4):356-366.

EGGLETON, P., BIGNELL, D.E., SANDS, W.A., MAWDSLEY, N.A., LAWTON, J.H., WOOD, T.G. \& BIGNELL, N.C. 1996. The diversity, abundance, and biomass of termites under differing levels of disturbance in the Mbalmayo Forest Reserve, Southern Camerron. Philos. Trans. R. Soc. Lond. B Biol. Sci. 351(1335):51-68.

ETTERSHANK, G., ETTERSHANK, J.A. \& WHITFORD, W.G. 1980. Location of food sources by subterranean termites. Environ. Entomol. 9(5):645-648.

FERRAR, P. \& WATSON, J.A.L. 1970. Termites (Isoptera) associated with dung in Australia. J. Aust. Entomol. Soc. 9:100-102. 
FONTES, L.R. 1979. Os cupins. Cien. Cult. 31(9):986-992.

FONTES, L.R. 1992. Key to the genera of New World Apicotermitinae (Isoptera: Termitidae). In Insect of Panama and Mesoamerica (D.A. Quintero \& A. Aiello, eds.). Oxford University Press, New York, p.242-248.

GILBERTSON, R.L. 1984. Relationships between insects and wood-rotting basidiomycetes. In Fungus-insect relationships (Q. Wheeler \& M. Blackwell, eds.). Columbia University Press, New York, p.130-165.

GRASSÉ, P.P. 1949. Ordre des Isoptères ou termites. In Traité de zoologie (P.P. Grassé, ed.). Masson, Paris, v.9, p.408-544.

GRASSÉ, P.P. 1986. Termitologia. Masson, Paris, Tomo 3.

GRASSÉ, P.P. \& NOIROT, C. 1959. L'évolution de la symbiose chez lês Isoptères. Experientia 15(10):365-372.

HARRIS, W.V. 1961. Termites: their recognition and control. Longmans, Green and Company, London.

HARRIS, W.V. 1970. Termites of the Palaeartic region. In Biology of termites (K. Krishna \& F.M. Weesner, eds.). Academic Press, New York, v.2, p.295-313.

HEWITT, P.H., WATSON, J.A.L., NEL, J.J. C. \& SCHOEMAN, I. 1972. Control of the change from group to pair behaviour by Hodotermes mossambicus reproductives. J. Insect Physiol. 18(1):143-150.

HIGASHI, M., ABE, T. \& BURNS, T.P. 1992. Carbon-nitrogen balance and termite ecology. Proc. R. Soc. Lond. B Biol. Sci. 249(1326):303-308.

HIGHLEY, T.L. \& KIRK, T.K. 1979. Mechanisms of wood decay and the unique features of heartrots. Phytopathology. 69(10):1151-1157.

JONES, D.T. \& GATHORNE-HARDY, F. 1995. Foraging activity of the processional termite Hospitalitermes hospitalis (Termitidae: Nasutitermitinae) in the rain forest of Brunei, north-west Borneo. Insectes Soc. 42(4):359-369.

KALSHOVEN, L.G.E. 1958.Observations on the black termites, Hospitalitermes spp., of Java and Sumatra. Insectes Soc. 5(1):9-30.

KRISHNA, K. 1969. Introduction. In Biology of termites (K. Krishna \& F.M. Weesner, eds.). Academic Press, New York, v.1, p.1-17.

LABOSKY JR., P. 1979. Chemical constituents of four southern pine barks. Wood Sci. 12(2):80-85.

LA FAGE, J.P. 1976. Nutritional biochemistry, bioenergetics, and nutritive value of the dry-wood termite, Marginitermes hubbardi (Banks). Ph.D. Dissertation, University of Arizona, Tucson.

LA FAGE, J.P. \& NUTTING, W.L. 1978. Nutrient dynamics of termites. In Production ecology of ants and termites (M.V. Brian, ed.). Cambridge University Press, Cambridge, p.165-232.

LEE, K.E. \& WOOD, T.G. 1971. Termites and soils. Academic Press, London.

LENZ, M. 1976. The dependence of hormone effects in termite caste determination on external factors. In Phase and caste determination in insects (M. Luscher, ed.). Pergamon Press, Oxford, p.73-89.

LENZ, M. 1994. Food resources, colony growth and caste development in wood-feeding termites. In Nourishment and evolution in insect societies (J.H. Hunt \& C.A. Nalepa, eds.). Westview Press, Boulder, p.159-210.

MARTIN, M.M. 1979. Biochemical implications of insect mycophagy. Biol. Rev. Camb. Philos. Soc. 54(1):1-21.

MARTIN, M.M. \& MARTIN, J.S. 1979. The distribution and origins of the cellulolytic enzymes of the higher termite Macrotermes natalensis. Physiol. Zool. 52(1):11-21.

MATHEWS, A.G.A. 1977. Studies on termites from the Mato Grosso State, Brazil. Academia Brasileira de Letras, Rio de Janeiro.

MEDEIROS, L.G.S. 2001. Variação temporal e espacial da atividade de Syntermes molestus e $S$. spinosus (Isoptera, Termitidae): base para avaliação de seu papel na decomposição da serapilheira em floresta de terra firme na Amazônia Central, Brasil. Tese de Doutorado, Instituto Nacional de Pesquisas da Amazônia, Universidade do Amazonas, Manaus.

MILL, A.E. 1984. Predation by the Ponerine ant Pachycondyla comutata on termite of the genus Syntermes in Amazonian rain forest. J. Nat. Hist. 18:405-410.
MIURA, T. \& MATSUMOTO, T. 1997. Open-air litter foraging in the nasute termite Longipeditermes longipes (Isoptera: Termitidae). J. Insect Behav. 11(2):179-189.

MIURA, T. \& MATSUMOTO, T. 1998. Foraging organization of the open-air processional lichen-feeding termite Hospitalitermes (Isoptera, Termitidae) in Borneo. Insectes Soc. 45(1):17-32.

NALEPA, C.A. 1994. Nourishment and the origin of termite eusociality. In Nourishment and evolution in insect societies (J.H. Hunt \& C.A. Nalepa, eds.). Westview Press, Boulder, p.57-104.

NOIROT, C. 1992. From wood- to humus-feeding: an important trend in termite evolution. In Biology and evolution of social insects (J. Billen, ed.). University of Leuven Press, Leuven, p.107-119.

PRESTWICH, G.D., BENTLEY, B.L. \& CARPENTER, E.J. 1980. Nitrogen sources for Neotropical nasute termites: fixation and selective foraging. Oecologia 46(3):397-401.

RATCLIFFE, F.N., GAY, F.J. \& GREAVES, T. 1952. Australian termites: the biology, recognition, and economic importance of the common species. C.S.I.R.O., Melbourne.

ROONWALL, M.L. 1970. Termites of the Oriental region. In Biology of termites (K. Krishna \& F.M. Weesner, eds.). Academic Press, New York, v.2, p.315-391.

RUDMAN, P. \& GAY, F.J. 1963. The causes of natural durability in timber. $\mathrm{X}$. The deterrent properties of some three-ringed carbocyclic and heterocyclic substances to the subterranean termite Nasutitermes exitiosus (Hill). Holzforschung 17(1):21-25.

RUDMAN, P. \& GAY, F.J. 1967a. The causes of natural durability in timber. $\mathrm{XX}$. The causes of variation in the termite resistence of jarrah (Eucalyptus marginata Sm.) Holzforschung. 21(1):21-23.

RUDMAN, P. \& GAY, F.J. 1967b. The causes of natural durability in timber. XXI. The anti-termitic activity of some fatty acids, esters and alchohols. Holzforschung 21(1):24-26.

SANDS, W.A. 1965. A revision of the termite subfamily Nasutitermitinae (Isoptera: Termitidae) from the Ethiopian region. Bull. Br. Mus. (Nat. Hist.) Entomol. Suppl. 4(1):1-172.

SANDS, W.A. 1969. The association of termites and fungi. In Biology of termites (K. Krishna \& F.M. Weesner, eds.). Academic Press, New York, v.1, p.495-524.

SANDS, W.A. 1972. The soldiersless termites of Africa (Isoptera: Termitidae). Bull. Br. Mus. (Nat. Hist.) Entomol. Suppl. 18(1):1-243.

SCHEFFRAHN, R.H. \& RUST, M.K. 1983. Drywood termite feeding deterrents in sugar pine and antitermitic activity of related compounds. J. Chem. Ecol. 9(1):39-55.

SLEAFORD, F., BIGNELL, D.E. \& EGGLETON, P. 1996. A pilot analysis of gut contents in termites from the Mbalmayo Forest Reserve, Cameroon. Ecol. Entomol. 21(3):279-288.

SNYDER, T.E. 1948. Our enemy the termite. Comstock Publishing Company, Ithaca.

TAYASU, I., ABE, T., EGGLETON, P. \& BIGNELL, D.E. 1997. Nitrogen and carbon isotope ratios in termites: an indicator of trophic habit along the gradient from wood-feeding to soil-feeding. Ecol. Entomol. 22(3):343-351.

THORNE, B.L. 1997. Evolution of eusociality in termites. Annu. Rev. Ecol. Syst. 28:27-54.

THORNE, B.L. \& KIMSEY, R.B. 1983. Attraction of Neotropical Nasutitermes termites to carrion. Biotropica 15(4):295-296.

WALLER, D.A. \& LA FAGE, J.P. 1986. Nutritional ecology of termites. In Nutritional ecology of insects, mites, spiders, and related invertebrates (F. Slansky Jr. \& J.G. Rodriguez, eds.). John Wiley \& Sons, New York, p.487-532.

WHITFORD, W.G., STEINBERGER, Y. \& ETTERSHANK, G. 1982. Contributions of subterranean termites to the "economy" of Chihuahuan Desert ecosystems. Oecologia. 55(3):298-302.

WOOD, T.G. 1978. Food and feeding habits of termites. In Production ecology of ants and termites (M.V. Brian, ed.). Cambridge University Press, Cambridge, p.55-80. 
WOOD, T.G. \& SANDS, W.A. 1978. The role of termites in ecosystems. In Production ecology of ants and termites (M.V. Brian, ed.). Cambridge University Press, Cambridge, p.245-292.

WOOD, T.G. \& THOMAS, R.J. 1989. The mutualistic association between Macrotermitinae and Termitomyces. In Insect-fungus interactions (N.
Wilding, N.M. Collins, P.M. Hammond \& J.F. Webber, eds.). Academic Press, London, p.69-92.

WOOD, T.G., JOHNSON, R.A., BACCHUS, S., SHITTU, M.O. \& ANDER SON, J.M. 1982. Abundance and distribution of termites (Isoptera) in a riparian forest in the southern Guinea savanna vegetation zone of Nigeria. Biotropica 14(1):25-39. 